\title{
Improving the Fit of Respiratory Face Masks through 3D Scanning, Finite Elements Analysis and Additive Manufacturing
}

\author{
Loïc DEGUELDRE*1, Jonathan BORDUAS ${ }^{*}$, Francis DION², \\ Patrick LAURIN ${ }^{2}$, Aude CASTONGUAY², Sean-Philippe VIENS², \\ Franck LE NAVÉAUX'1 ${ }^{\text {, Bahe HACHEM }}{ }^{1}$, David BENOIT ${ }^{1}$, Julien CLIN ${ }^{1}$ \\ ${ }^{1}$ Numalogics, Montréal (QC), Canada; \\ 2 Technologies ShapeShift 3D Inc., Montréal (QC), Canada \\ https://doi.org/10.15221/20.33
}

\begin{abstract}
The 2020 COVID-19 pandemic sheds a new light on the importance of wearing a mask to prevent virus propagation. A respiratory mask's efficiency is highly dependent on its adjustment with the face. With traditional face masks, achieving a perfect seal with the face proves to be challenging, even impossible without the aggressive tightening of its straps. Healthcare workers typically wear protective face masks for several consecutive hours, leading to discomfort, inflammation, or more serious injuries.

This paper highlights how the combination of current technologies such as 3D scanning, Finite Element simulation, machine learning, and additive manufacturing offers a seamless workflow to generate a sterilizable, reusable and validated custom-fit mask.

The process starts with a 3D face scan acquired from a mobile 3D scanning device. From this scan, the software Shapeshift3D automatically repairs the 3D scan and fits the mask to the facial features. A custom Finite Element model is created by morphing the mesh of a generic face model and its corresponding mask onto the person's face. The fit, achieved through customization, is then assessed via biomechanically simulating the mask's tightening while accounting for the face-mask interface and the facial tissue's behavior. A real time assessment of the mask's sealing properties and the pressure on the skin is made possible by a machine learning algorithm that was trained on a database of face mask tightening simulations (ANSYS software). This approach allows to quantify in real-time the level of fit achieved through customization. The custom mask is then 3D printed and assembled before being shipped to the end-user.
\end{abstract}

Keywords: 3D Face Scan, 3D scanning, custom fit, Additive Manufacturing, Machine Learning, Finite Element Analysis, Real-Time Simulation, Cloud Computing, Biomechanics, Mesh Morphing.

\section{Introduction}

The year 2020 started with an outbreak of severe acute respiratory syndrome coronavirus 2 (SARSCoV-2)[1], that first occurred in the Wuhan province in China back in December 2019 but soon spread all over the world. On March 11th, it was declared a pandemic by the World Health Organization (WHO). As of today, according to WHO, more than 25 million cases of the coronavirus disease (COVID-19) have been confirmed causing more than 800,000 deaths [2]. Undeniably, the virus is highly contagious and is known to spread among people through close contact and/or via respiratory droplets generated when speaking, coughing or sneezing within a distance inferior to $1.6 \mathrm{~m}[3,4,5]$. This virus could be airborne as well [6].

Therefore, to prevent virus transmission, WHO recommends implementing various control measures to break chains of transmission such as physical and social distancing, 14 days quarantine for infected people, and encourage the use of fabric face masks in public places [7]. In clinical areas, health workers and caregivers shall also use medical masks such as N95, FFP2 and FFP3 respirators during their daily routines [7].

Several studies demonstrate the performance of N95 respirators as far as their filtration efficiency is concerned $[8,9]$. However, to be fully efficient in preventing COVID-19 infections, respirator masks must meet several criteria set by the National Institute for Occupational Safety and Health (NIOSH) among which the respirator must fit the user's face snugly (i.e., create a seal) to minimize the number of particles that bypass the filter through gaps between the user's skin and the respirator seal [10].

\footnotetext{
* LDegueldre@numalogics.com; numalogics.com

* Jonathan.Borduas@shapeshift3D.com; shapeshift3D.com
} 
Fit testing and seal checking are therefore critical to ensure no leakage occurs. However, good fit and adequate sealing may be hard to obtain with generic masks without applying an important tightening force to the mask straps. Indeed, several health care providers who must wear respiratory mask all day long reported facial pressure injuries and pictures of such wounds were widely shared on the social media (Figure 1). Pressure ulcers over the bony prominences of the face have been reported with the usage of non-invasive ventilation face mask, while pressure ulcers over the dorsum of the nose are associated with wearing N95 respirators during extended period of time [11].

A study provided an explanation to this phenomenon, reporting that health care workers who suffered from pressure ulcers had secured their respiratory mask tighter than required - fearing contracting the COVID-19 due to an imperfect seal [11].

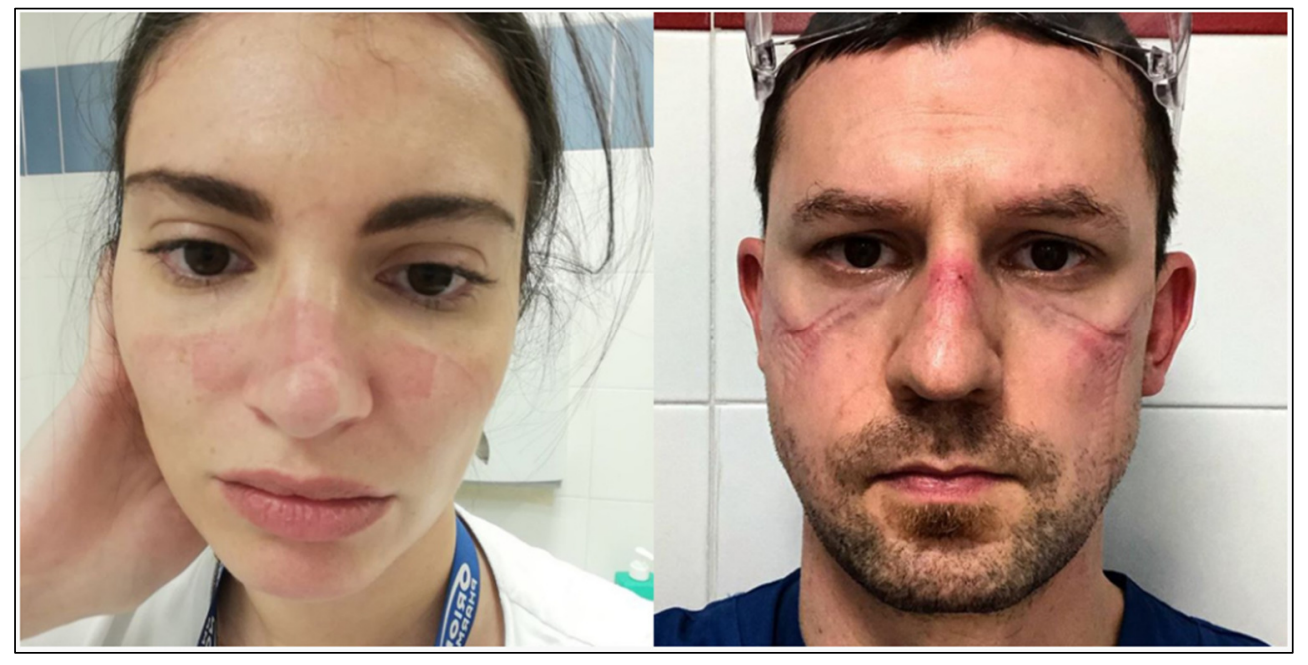

Figure 1: Facebook/Martina Benedetti/Nicola Sgarbi

One solution to ensure a proper seal would be to provide a custom fit mask rather than generic masks that aim at fitting a maximum of faces shapes. Current innovation and technology such as 3D scanning and $3 \mathrm{D}$ printing are revolutionizing virtual design and mass customization of wearables. However, designing a custom fit mask based on someone's 3D-scanned face may not ensure optimal sealing since the flexible nature of the mask and the face's soft tissues may alter the fit when tightening the mask. Understanding the biomechanical interaction between a mask and the face's soft tissues could help in designing a better mask and have a better estimation of the resulting fit.

In this paper, we present a technological workflow allowing to virtually design custom-fit masks and asses the fit with the customer's face (Figure 2). The first step starts with a user's 3D facial scan using a dedicated application on a smartphone. The scan is then used to morph a generic mask design to perfectly fit the user's face. The scan is also used to build a finite element model aiming at simulating the interaction of the custom-mask with the user's face. Once validated, the customized geometry is finally ready to be 3D printed and the mask is eventually sent to the customer along with the simulationpowered heat map of expected pressure and gap with the user's face. The last section of this paper describes the ongoing work using Artificial Intelligence $(\mathrm{Al})$ to replace the simulation and provide the fit assessment result in real-time to engaging the user and confirming that the product will fulfill their needs.

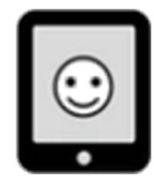

SCAN

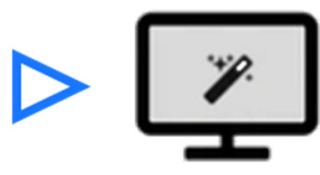

FIT

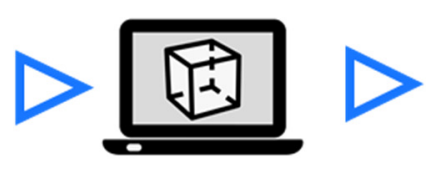

SIMULATE

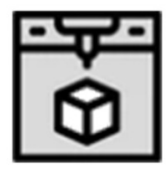

PRINT

Figure 2: Seamless workflow to get a custom BeyondFit ${ }^{T M}$ Mask. 


\section{From Face Scanning to Custom-Fit Mask}

Although the process of custom-fitting is scan-agnostic and could therefore use any scanning technology, not all technologies are user-friendly enough to guarantee the scan's quality, regardless of the user's training. The solution to this was to develop the BeyondMask ${ }^{\mathrm{TM}}$ mobile app [12] using the ARCore scanning technology for Android phones [13], and the ARKIT scanning technology for Apple Inc. products [14]. This allows the user to get a 3D scan of their face from one single picture, therefore reducing user-based bias on the resulting scan. Figure 3 shows an example of face scans using both technologies.

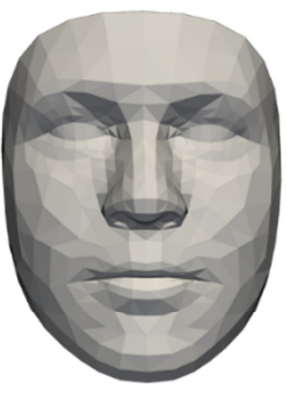

(a) BeyondMask Android

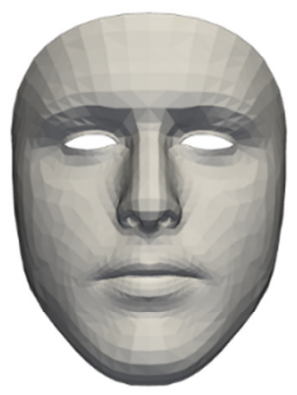

(b) BeyondMask iPhone

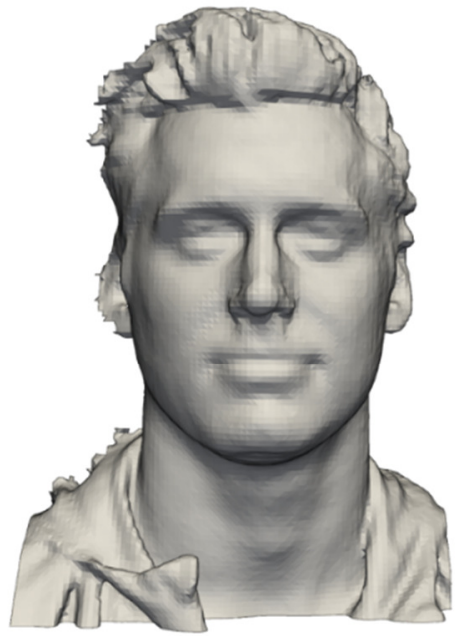

(c) 3DSizeMe

Figure 3: Resulting face scans using the BeyondMask ${ }^{T M}$ mobile app on a) Android; b) iPhone; c) 3DSizeMe

When scans are taken through the BeyondMask ${ }^{\mathrm{TM}}$ app, the rest of the jaw and the neck are extrapolated using the Flame Al database [15].

Shapeshift 3D's proprietary algorithms can also adapt to other scanning technologies, such as 3DSizeMe (TechMed3D, Québec, QC). This application however requires a specific scanning device to be added to an iPad, as well as a trained user to scan the client.

Once the scan is taken, the morphing of the mask onto the face is done through 4 main steps, based on Shapeshift 3D's Constraint Template Morphing (CTM) modelling paradigm: scan landmarking, scan reconstruction, mask positioning, and mask fitting.

Scan landmarking consists of automatically detecting 73 anatomical and non-anatomical landmarks on the face, using a Deep-MLVM algorithm [16]. The scans are then reconstructed by cleaning, scaling, remeshing, filling holes, symmetrizing, and smoothing [17]. While those operations could introduce additional bias to the scan, we believe they greatly help reducing user-based bias and result in smoother more comfortable masks with better aesthetics. The initial positioning of the mask is done by placing the outline on the reconstructed scan, based on the 73 landmarks. The mask is then fitted on the reconstructed scan by placing all sections requiring specific constraints, such as roto-translations only or perfect-fit to the surface, and then morphing the whole mask according to those constraints. All this process runs automatically and requires no user intervention, using the CTM modelling paradigm unique to Shapeshift 3D. Figure 4 shows the output of each of the four steps using both a scan taken using the BeyondMask ${ }^{\mathrm{TM}}$ app on iPhone, and a scan using the 3DSizeMe app. 

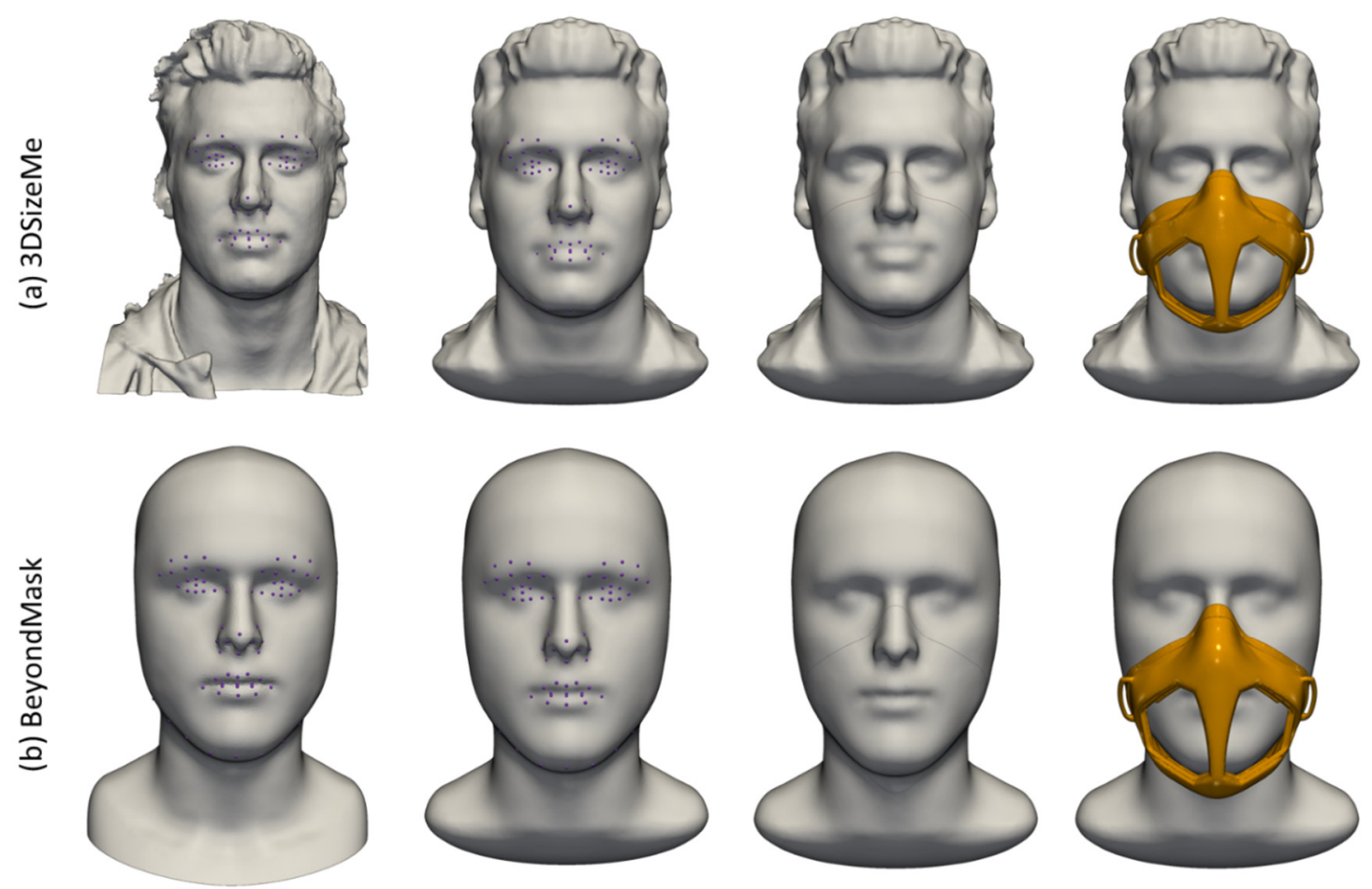

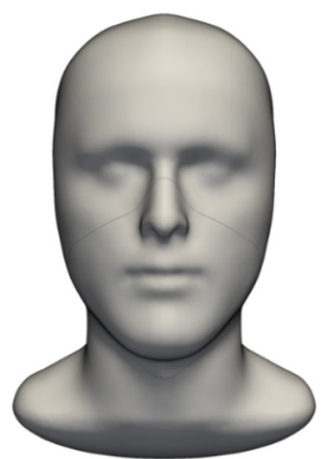

Positioning

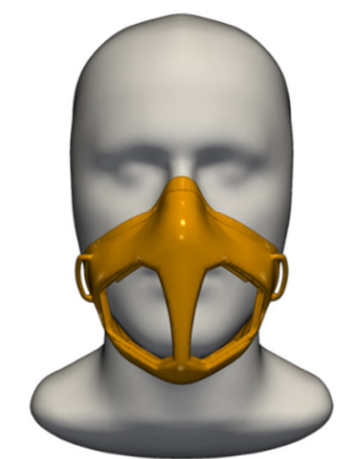

Fitting

Figure 4: Outputs of scan landmarking, scan reconstruction, mask positioning and mask fitting for a scan taken with a) 3DSizeMe; b) BeyondMask ${ }^{T M}$ on iPhone

The customized masks follow the initial mechanical requirements, such as minimal thickness and the shape and size of certain pieces. As shown in Figure 5, the strap holders, to the left and right, maintain the right size and shape, while the filters' housing is not deformed at all, simply positioned appropriately regarding to the scan. Some other sections of the masks, such as the male and female parts of the silicone locking system, are not deformed either as to guarantee sealing and ease of assembly.

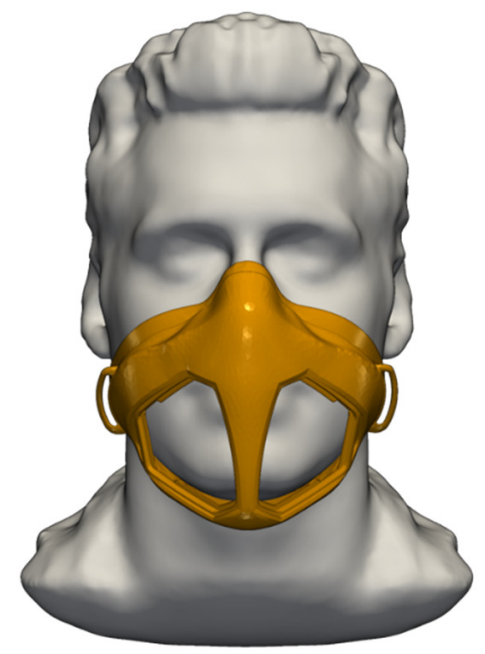

(a) Frontal view

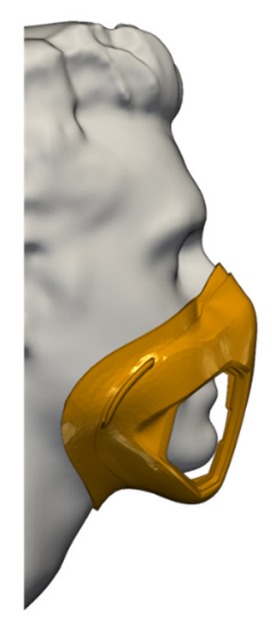

(b) Lateral view

Figure 5: a) Front view; and b) lateral view of a BeyondFitTM mask 


\section{Mask Sealing Assessment: Simulating the Biomechanics of the Face-Mask Interaction}

\subsection{Advantages of using Finite Element Analysis}

For many biomedical applications, it is practically impossible to test devices in vivo, due to the nature of the device itself. For instance, you cannot conduct implant testing onto a human body. It is all the truer when dealing with patient specific devices since each device is unique. Essentially, numerical simulation in general and, in particular, Finite Element Analysis (FEA) can play a major role.

FEA is a computer-based method widely used by engineers to model complex assemblies for many decades. The Finite Element Method basically consists in dividing the domain of interest into simpler parts: the elements. This discretization leads to a collection of equations that represent each subdomain behavior with respect to a loading condition with accounting its material properties. Breaking down a complex and large structure into smaller sections helps in understanding how the overall assembly will sustain external stimuli.

To assess the sealing of the BeyondFit ${ }^{\mathrm{TM}}$ mask on any face, a generic Finite Element Model is created on an assembly of a generic face with its corresponding mask. Then, to give the specific facial pressure contour, the mask is automatically morphed onto the person's face based on their scan prior to simulating strap tightening. Among the plethora of candidate commercial software, ANSYS (ANSYS Inc., Pittsburg, USA) is chosen to run mask tightening simulations.

The following subsections describe the chosen assumptions to set up the generic assembly.

At the beginning of the pandemic, marketing papers [18] provided more complex ways to analyze N95 respiratory mask performance or behavior. Indeed, breathing and respiratory droplets flow can be simulated, and it can be seen that wearing a mask can prevent them from propagating. However, it is a highly complex simulation, accounting for fluid-structure interactions. Moreover, the facial pressure value due to the mask's tightening can be linked to the comfort associated with wearing the mask [19] and can be used to assess leakage occurrence, or the lack thereof. Accordingly, we focused our study on the biomechanical behavior of the mask on the face.

\subsection{Simulation Assumptions}

\subsubsection{Mask Model}

In order to speed-up simulation time without reducing the quality of the model, the initial geometry of the generic mask was simplified for the purpose of the analysis. The FEM of the mask was thus based on linear shell elements (ANSYS SHELL181 elements) which are commonly used and well suited to represent thin structures (Figure 6). A mid-surface extraction was performed on the silicon shell. Polyamide being far stiffer than silicon and facial soft tissues, only the outer surface of the mask needed to be kept to solely represent the stiffness of the whole mask. This allowed to get rid of many details that would have made the model too complex (cf. inside view of the mask in Figure $6 \mathrm{a}$ ).

Hence, the mask mesh was made of 4 parts: the silicon shell, the overall mask and the left and right grids that are clamped on the mask to retain filters. Filters were not included in the model as they have negligible impact on the mechanical behavior of the assembly. 


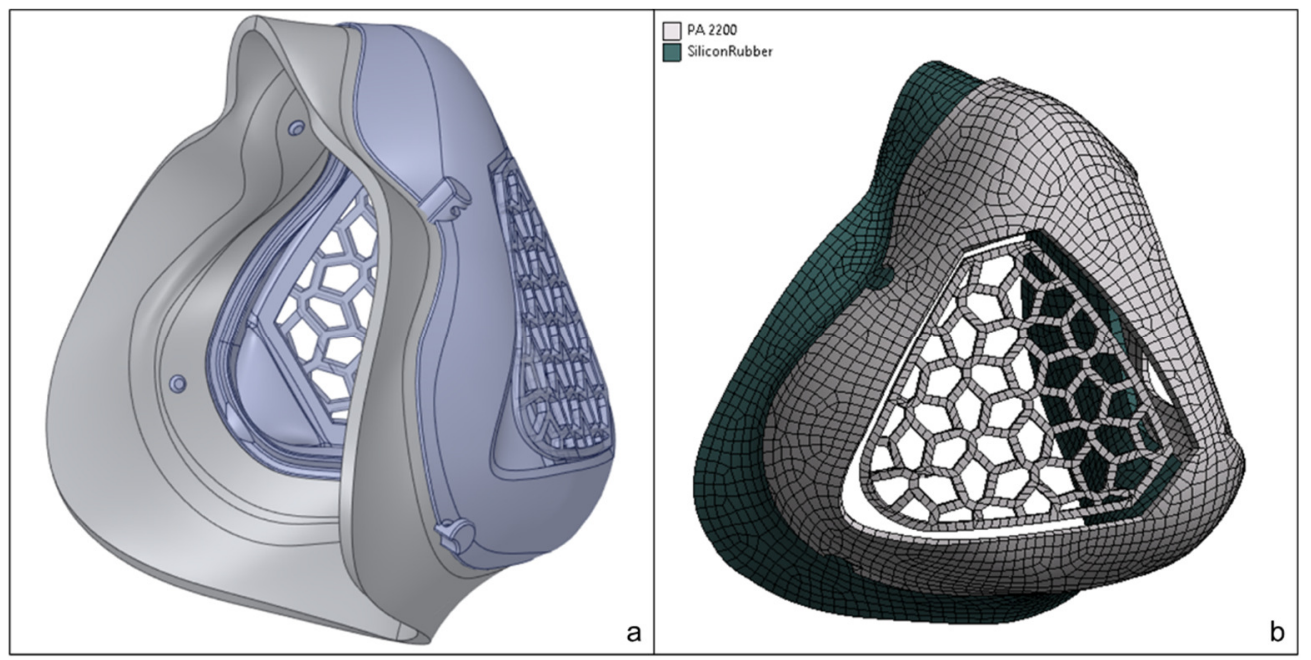

Figure 6: a) Detailed view of the generic mask geometry; b) Corresponding simplified mesh to be used in the FEA

Each grid was restrained from moving apart from the mask by a fixed joint connecting the grid to the overall mask.

To simulate the connection of the silicon shell on the polyamide part of the mask, a bonded contact was defined, so that the two materials cannot move apart from each other (Figure 7).

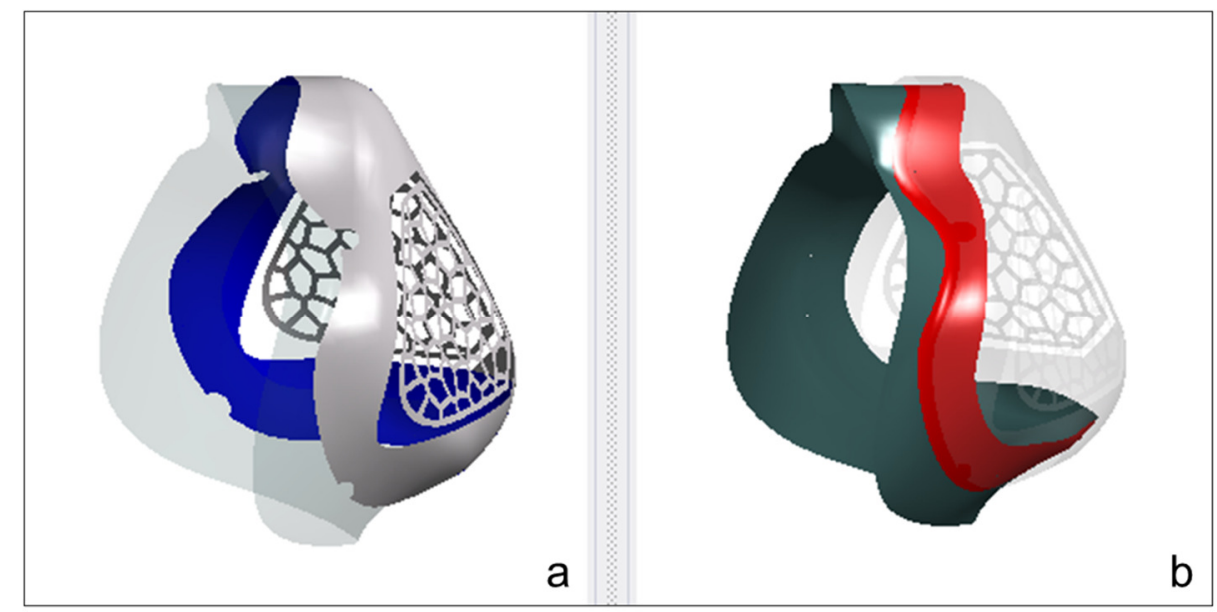

Figure 7: Connection between mask polyamide (a) and silicon (b) shells.

As far as the material properties are concerned, standard linear elastic properties provided by the polyamide manufacturer were used. As for the silicon shell, a non-linear material was used. Indeed, uniaxial tension-compression tests were performed on silicon samples to get an accurate understanding of its behavior. The results of these experimental tests led us to represent it with a hyper elastic material using a $3^{\text {rd }}$ order Yeoh model.

\subsubsection{Face Model}

The main issue of the face model is to represent all the facial soft tissues in a simple and generic way, appropriate to represent any person. The starting point is a three-dimensional anatomically accurate model of the head (Figure 8a, Zygotebody, Zygote Media Group Inc, American Fork, UT).

A cross section of the head would reveal that you may find fat tissues, muscles, ligaments, tendons, blood vessels and bone underneath the skin. It would be quite complex to model all these materials and their interaction with each other. Thus, the chosen approach is to create a global and unique homogenized material that would represent them all (Figure 8b). To reduce the model size, the head is reduced to the face from forehead to neck and the stiffness of the skull bones is modeled as a boundary condition (see Section 3.2.4) 
The mechanical behavior of this material is derived from a numerical model of the human body (numa, Numalogics, Montréal, QC) in which soft tissues are modeled with a hyper elastic material using a $2^{\text {nd }}$ order Yeoh model [20].

Finally, a volumetric mesh is generated using tetrahedron quadratic elements - ANSYS SOLID187 elements (Figure 8c).

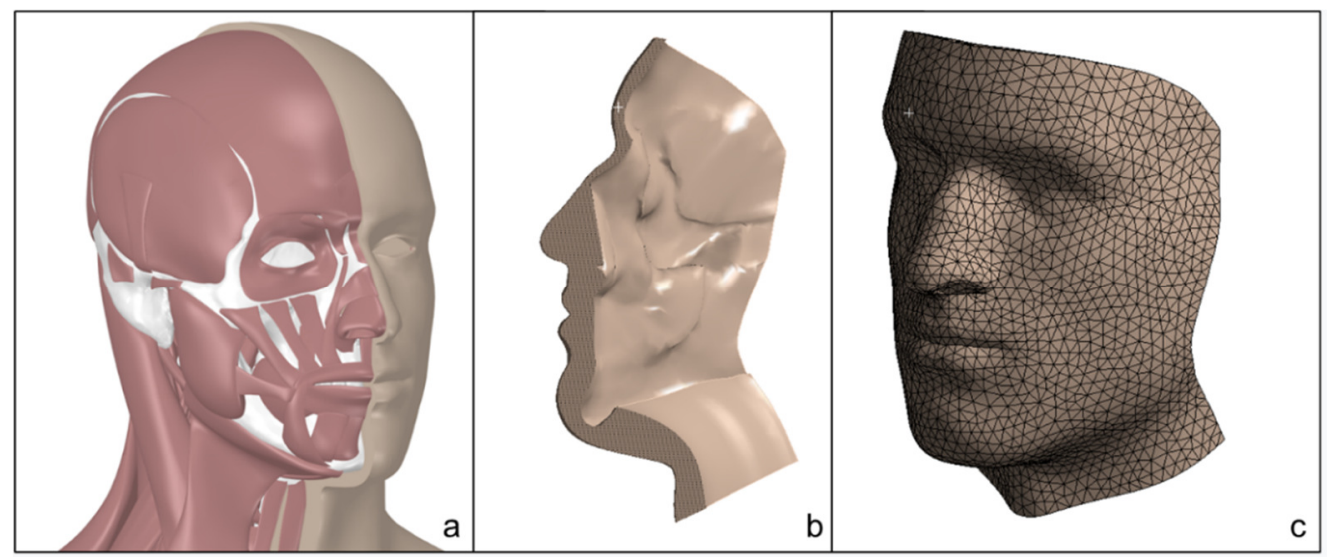

Figure 8: Face Finite element Model Generation: a) Starting Geometry;

b) Homogenized soft tissues (Cross Section View); c) Mesh

\subsubsection{Face-Mask Interaction}

In ANSYS, interactions between parts are modeled through contact regions for which formulation and properties can be set to better represent the interface behavior. Frictional contact was chosen as it best represents the physical behavior of the face-mask interface. Several studies [21, 22] show that the skin's coefficient of friction not only depends on the lubricating condition of the skin and body zones but also on the contacting material. To model the contact of the silicon shell on the face skin (Figure 9), a coefficient of friction of 0.51 was used in our model, reflecting an average value of the reported literature [23]. Pure penalty method was used for the contact formulation, which required the definition of the contact stiffness. Its value was set to $0.19 \mathrm{~N} . \mathrm{mm}^{-2}$ through the definition of a contact stiffness factor (FKN) constant value of 1 during the whole simulation process. This value has been set after a convergence study, which ensured that it did not affect results accuracy while maintaining a decent simulation time.

The contact detection was set at nodal point using the surface projection-based method which ensures more uniform contact results.

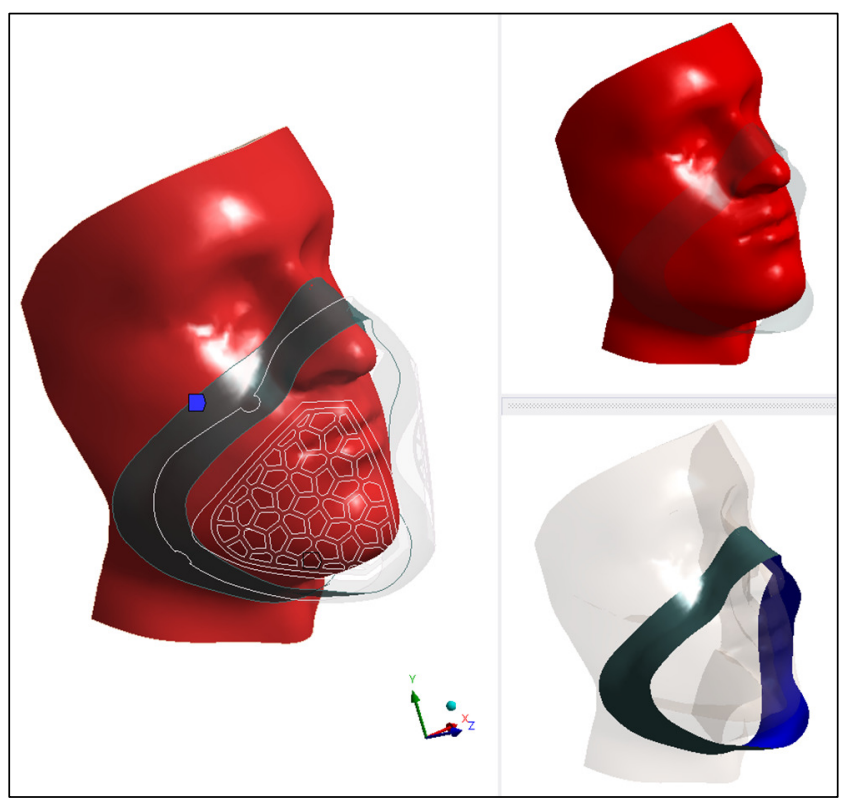

Figure 9: Face-Mask Contact Region 


\subsubsection{Loading and Boundary Conditions}

To mimic the tightening of the mask over the face, a force of $20 \mathrm{~N}$ is applied on the polyamide parts of the mask. Both the direction of the force and its magnitude are evaluated based how mask straps are physically tightened (Figure 10).

The neck's bottom surface (Face D in Figure 10) and the cutting surfaces at the back of the head (Face $C$ in Figure 10) are restrained from moving in all directions, so that the head does not move when applying the mask tightening force.

All the mesh nodes of the back surfaces of the face's soft tissues (Faces A in Figure 10) are restrained from moving in the normal direction but free to move in the tangential direction with respect to the back surfaces of the face that are contacting the skull bones (a frictionless support has been defined to achieve this behavior). Such an interface allows a small sliding of the soft tissues' nodes on the bones surface.

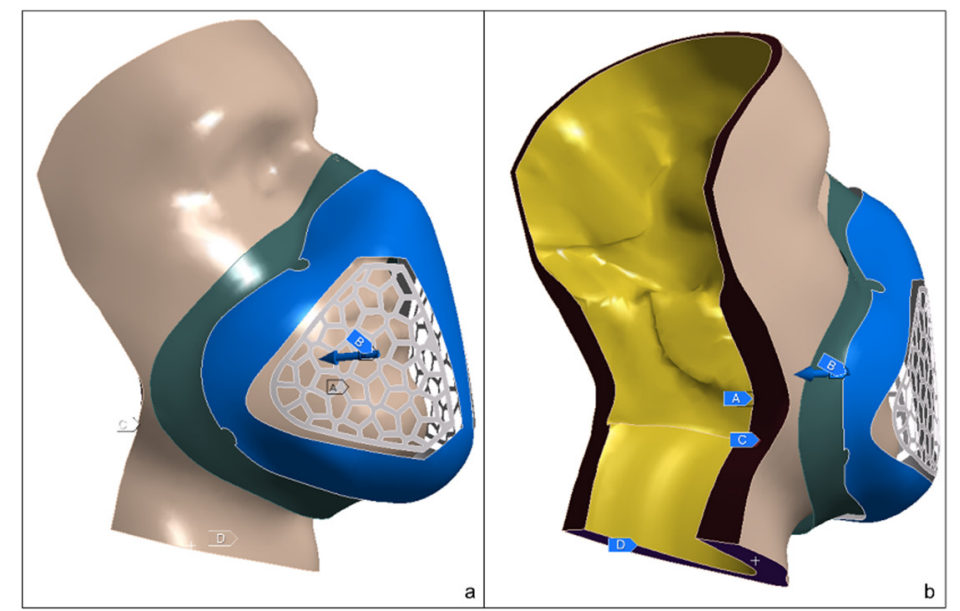

Figure 10: a) Loading Force on the mask and b) Boundary conditions on the back of the face

\subsubsection{Analysis Settings}

Inertial and transient effects were not considered in the mask tightening simulations. The result of interest is the equilibrium state at the end of the strap tightening application. Thus, the assembly was simulated in an iterative non-linear static structural environment, with loading force being ramped gradually up to its magnitude of $20 \mathrm{~N}$

During the whole simulation process the non-linearities due to large displacements, large deformations and status change in the contact elements were accounted for using the Newton-Raphson method. The convergence criterion of the non-linear process is set to a maximum unbalance force of $1 \mathrm{~N}$.

\subsection{Preliminary Results on the generic model}

\subsubsection{Total Deformation}

Figure 11 shows the total displacement resulting of the mask tightening. On the front view (a), displacement is higher on the right side of the mask, since before tightening, the mask is not perfectly in front of the face: it is automatically adjusted by a small rotation with the tightening force, leading a slightly higher displacement for that portion of the mask. The cross-section view (b) shows how well the silicon shell is strained to perfectly fit the face on the top of the nose and under the chin. Figure 11(c) shows the deformation of the skin with a hotspot under the chin due to a sharp edge of the silicon shell. 


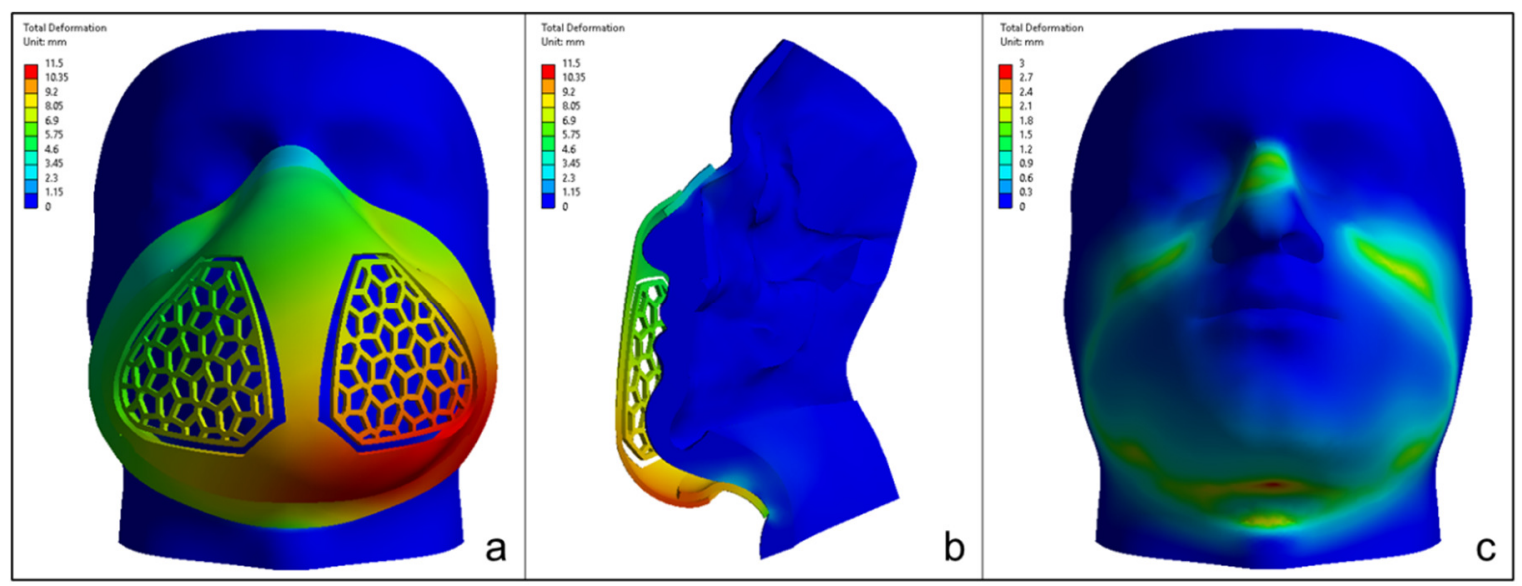

Figure 11:Total Deformation: a) Front View b) Cross-Section View c) Face Deformation

\subsubsection{Sealing}

Figure 12 shows the resulting gap between the silicon shell of the mask and the skin. The blue regions represent zones where the distance between the silicon and the face is less than $0.5 \mathrm{~mm}$.

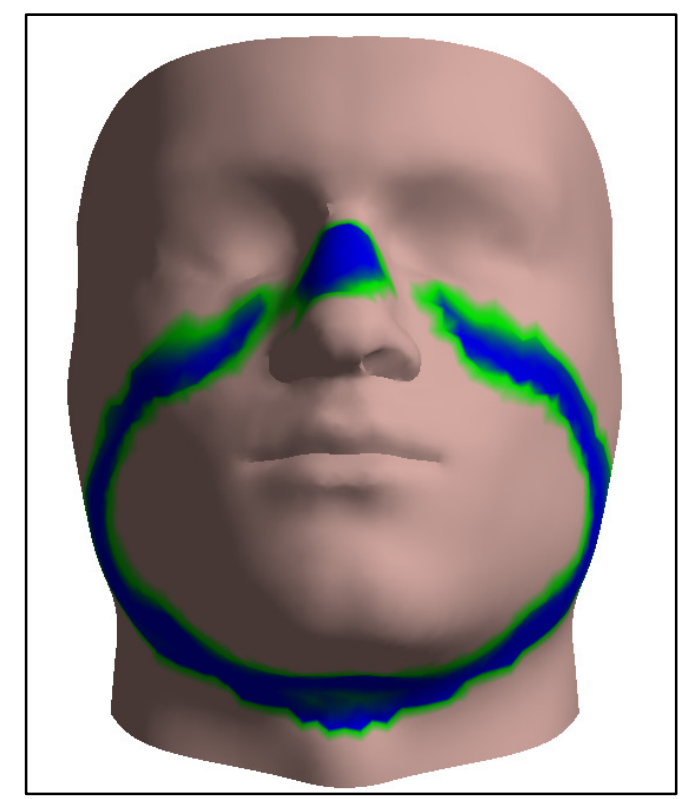

Figure 12: Gap between silicon shell and skin (blue color: Gap $<=0.5 \mathrm{~mm}$ )

\subsubsection{Contact Pressure Heatmap}

Figure 13 displays the distribution of the contact pressure when the mask is tightened on the face. The pressure is quite homogeneous without hotspots that would make the user uncomfortable. However, two regions show higher pressure:

- A zone over the nose dorsum, which is the region of the model where soft tissues are the least thick.

- Under the chin, which can also be explained by the sharp edge of the silicon shell contacting the neck skin (see deformation results in Section 3.3.1 and Figure $11 \mathrm{c}$ ) 


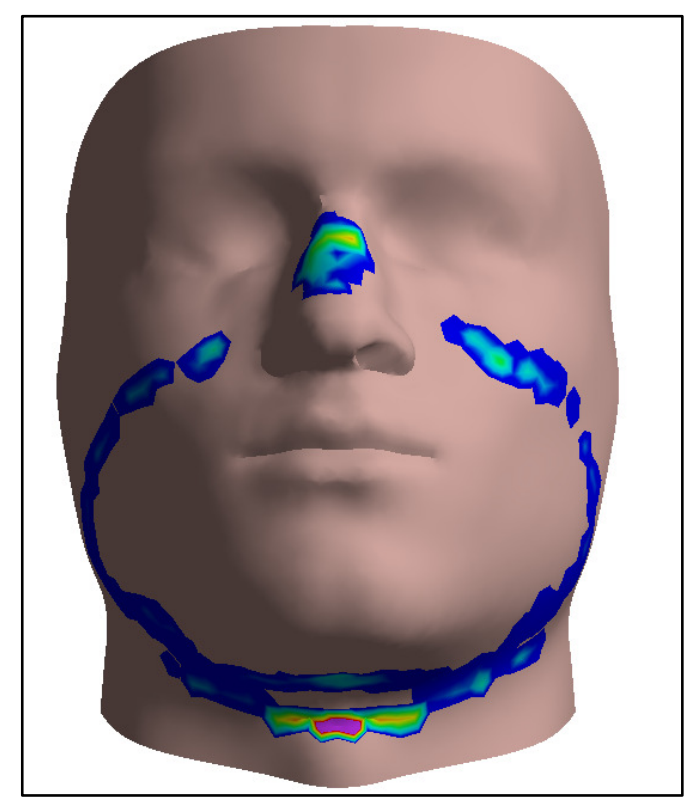

Figure 13: Contact Pressure

\subsection{Automatic Morphing}

The previous subsections described the way the FEA simulation was setup for the generic version of the mask. However, the goal is to analyze the custom fit mask that is morphed onto any people's face based on their scan. Hence, given the generic assembly created for the generic version, a Pythoncoded routine is developed to be able to automatically update the generic simulation with new face and mask meshes output from the morphing software. For each specific people, the mask contour pressure heat-map over the face is thus an automatic outcome of this simulation.

\section{Perspectives}

\subsection{Biomechanics simulation for marketing and certification}

The pressure heat-map given by the FEA is a great outcome to engage the customer and give them a tool to help them make an informed choice. Such numerical tools could help customers to easily compare the performance of a generic mask to a custom fit mask from a sealing and facial pressure perspective. Strap tightening simulations results can be readily obtained and compared for a generic and custom-fit mask for an individual's face. With both pressure heatmaps readily accessible, a customer could anticipate the comfort feeling from both masks for an objective choice to buy the best one.

On another level, the pressure heat map could help in getting the N95 certification delivered by the $\mathrm{NIOSH}$ for the US market or the CAN95 certification given by Health Canada for the Canadian domestic market. As a matter of fact, both certifications require physical testing. If those tests can easily be implemented for large scale production of respirators, it is almost impossible to have it done for every single custom mask.

To meet the certification requirements without physical testing a rational could be used based on simulation results. Physical testing on the generic version of the mask needs first to be performed. Once this generic version of the mask is approved, it becomes a predicate for the custom version. Then, virtual testing could demonstrate that:

- The simulated gap between the face and the mask, once the straps tightened, would be minimized when using the custom-fit mask compared to the generic one.

- When the custom-fit mask is tightened properly on the face, the contact pressure simulated on the face would be at least as good as the generic one.

Having simulation showing that the custom version of the mask is at least as good as the approved predicate, any custom mask could finally be approved. 


\subsection{Artificial Intelligence for real-time results}

To get real time pressure heat map, simulation results could be used in a machine learning (ML) algorithm. ML is a field of Artificial Intelligence (AI) in which algorithms make predictions without being explicitly programmed to do so [24]. Those predictions are based on a database of sample data, known as the training data. The training data are a set of example inputs associated with their desired outputs. The aim of the ML algorithm is to build a mathematical model that maps inputs to their corresponding outputs. The general rule that the algorithm has learnt will then be used to predict unknown outputs for inputs that have never be 'seen' by the algorithm.

In our case, the training dataset will consist in a set of face scans with associated custom mask and applied tightening force as input data and corresponding facial deformation and pressure map generated via FEM simulations as matching output.

To build the model's database, an initial cohort of 100+ input data with matching output results will be processed and randomly divided into $80 \% / 10 \% / 10 \%$ for training, validation, and testing respectively. The training dataset will serve to create three different $\mathrm{ML}$ models based on separate $\mathrm{ML}$ algorithms. The validation dataset is then used to evaluate the performance of each model and isolate the best candidate. Predicted pressure maps and skin deformation will be contrasted with their simulation counterparts to assess the ML model's performance and the prediction error. Finally, the testing set will serve to evaluate the candidate model's performance.

Subsequently, a real-time pressure map will be generated with the selected model via any mobile device capable of facial scanning. Moreover, each new scan will also serve to augment the training/validation dataset and fine-tune the ML model's hyperparameters and performance until deemed reliable with no added benefit in its effectiveness. At this stage, the ML model is frozen and will be used as the goldstandard for real-time pressure map prediction.

Such a seamless ML integration reassures the user's custom mask attains the best fit possible prior to purchasing.

\section{Conclusion}

In this paper, we demonstrated that we could combine some of the latest technologies in order to create a seamless workflow to virtually design custom-fit masks with assessing the fit to the customer's face. We are all the more confident of the success of this workflow as we recently (mid-October 2020) reached an important milestone since a batch of masks for early adopters is about to be 3D printed.

Now that the workflow is suitably setup, some more work still needs to be done for validating the process automation, improving scan acquisition precision, mask fitting and mesh morphing quality, checking the accuracy of the finite element model and building the database the ML algorithm will be trained on.

Finally, we strongly believe that this workflow could pave the road for other custom product developments whatsoever, with accounting for the human-device interaction's biomechanics in regulated fields.

\section{References}

[1] Gorbalenya AE, Baker SC, Baric RS, de Groot RJ, Drosten C, Gulyaeva AA, et al. (March 2020). "The species Severe acute respiratory syndrome-related coronavirus: classifying 2019-nCoV and naming it SARS-CoV-2". Nature Microbiology. 5 (4): 536-544. doi:10.1038/s41564-020-0695-z.

[2] "How COVID-19 Spreads". U.S. Centers for Disease Control and Prevention (CDC). 27 January 2020. Archived from the original on 28 January 2020. Retrieved 29 January 2020.

[3] Edwards E (25 January 2020). "How does coronavirus spread?". NBC News. Archived from the original on 28 January 2020. Retrieved 13 March 2020.

[4] WHO Coronavirus Disease (COVID-19) Dashboard, https://covid19.who.int/

[5] Anfinrud P, Stadnytskyi V, Bax CE, Bax A (May 2020). "Visualizing Speech-Generated Oral Fluid Droplets with Laser Light Scattering". The New England Journal of Medicine. 382(21): 20612063. doi:10.1056/NEJMc2007800. PMC 7179962. PMID 32294341. 